\title{
Ant Colony Optimization based Modified AOMDV for Multipath Routing in MANET
}

\author{
Chintan Kanani \\ M.Tech (IT) \\ Dept. of I.T., T.I.T. \\ Bhopal (M.P.), India
}

\author{
Amit Sinhal \\ M.Tech (IT \& SS), Ph.D \\ Dept. of I.T., T.I.T. \\ Bhopal (M.P.), India
}

\begin{abstract}
In this paper, modified ad-hoc on-demand multipath distance vector (AOMDV) for multipath routing using ant colony for mobile ad hoc networks (MANETs) is presented. For this purpose, Ant-AODV is used for comparison with AntAOMDV. The idea behind the working of Ant-AODV and Ant-AOMDV is that the RREQ message packets are sent to single path in case of Ant-AODV based routing and to multiple paths in case of Ant-AOMDV based routing. RREQ message packets can be termed as pheromone in terms of standard algorithm of ACO used by the ants. Selecting the transmission path dynamically through regular updating of pheromone of transmission path expects to improve routing performance. Simulation results show that Ant-AOMDV algorithm outperforms Ant-AODV effectively in terms of packet delivery fraction, normal routing load and packet drop compared with AODV and AOMDV. The main goal is to reduce the routing overhead, congestion and increase the performance.
\end{abstract}

\section{General Terms}

Security, Algorithms, Quality of Service, Routing, Load Balancing, Congestion control.

\section{Keywords}

MANET, Ant Colony Optimization, AODV, AOMDV, Pheromone.

\section{INTRODUCTION}

A Mobile Ad hoc network (MANET) is a set of mobile nodes which communicate wirelessly over radio frequencies with no centralized infrastructure. This is in stark contrast to the infrastructure of other networks such as Local Area Networks, and peer to peer networks. The properties of wireless communication and mobility resent huge problems to the creation of such networks and the maintenance of services on these networks. As the nodes keep moving away or towards each other, it is understandable that parameters like Quality of Service (QoS) will suffer unless special schemes are developed to sustain such networks [1]. With the ubiquity of laptops and wireless devices, there is a need of for the realization of such networks.

Routing is one of the most complicated problems to solve as ad hoc networks have a seamless connectivity to other devices in its neighborhood. Because of multi hop routing no default route is available. Each node acts as a router and forwards each other's packets to enable information sharing between mobile nodes. A MANET routing algorithm should not only find the shortest path between the source and the destination, but the algorithm should also be adaptive, in terms of the changing state of the nodes, the varying load conditions of the network and the varying state of the environment. Due to this reason, majority of MANETs are connection less in nature, since connections are less effective in delivering the QoS that is required in the rapidly changing MANET environment and impose additional overhead on the network [1]. MANETs are also multi-hop in nature, in that packets need to be relayed through other nodes to get to the destination. Thus MANETs require that traditional algorithms be redefined to accommodate these additional requirements.

To explore routing protocols in MANET, there are single path and multi path based routing protocols. AODV is one of the efficient protocols for single path based routing and AOMDV is suitable for multipath based routing. But, still AODV and AOMDV have its own drawbacks. The existing single path and multipath routing protocols have not fully used the route stability to carry on the routing. In this paper a new approach is presented for an on demand ad-hoc routing algorithm, which is based on ad-hoc on-demand multipath distance vector (AOMDV) and ant colony optimization. The aim is to find more stable paths so that the packet drop frequency can be decreased and the network performance will be improved. The remainder of this paper is organized as follows. In section 2 below a brief review of the related work is presented. In section 3 routing algorithms ACO-AODV and ACO-AOMDV are presented in detail with its advantages and problems. Subsequently, in section 4 some simulation results are presented to show the ability of the approach. Finally, a conclusion is given in section 5 .

\section{BACKGROUND}

This section describes routing protocols in MANET, existing standard routing protocols in single path and multipath routing, and general description of ant colony optimization and its usage in MANET.

\subsection{Routing Protocols}

Since the advent of DARPA packet radio networks in the early 1970s, various protocols have been developed for mobile ad-hoc networks. Such types of protocols must deal with the classic limitations of these networks, which include high error rates, high power consumption and low bandwidth. These routing protocols may be categorized as: (a) tabledriven and (b) source-initiated on-demand driven [2].

\subsubsection{AODV Protocol}

Ad hoc On-Demand Distance Vector (AODV) is a routing protocol for mobile ad hoc networks (MANETs) and other wireless ad hoc networks. It is a reactive protocol which means that it establishes a route to a destination only on demand. In comparison, the most common routing protocols of internet are proactive, which means that they find routing paths independently of the usage of the paths. AODV is a distance-vector routing protocol. It avoids the counting-to- 
infinity problem of other distance-vector protocols by using sequence numbers on route updates. It is capable of both unicast and multicast routing. The main advantage of this protocol is having routes established on demand and that destination sequence numbers are applied to find the latest route to the destination. The connection setup delay is lower. Its disadvantage is multiple RouteReply packets in response to a single RouteRequest packet can lead to heavy control overhead. Other disadvantage of AODV is unnecessary bandwidth consumption due to periodic beaconing. [3]

\subsubsection{AOMDV Protocol}

Ad hoc On-Demand Multipath Distance Vector (AOMDV) shares numerous characteristics with AODV. It is based on the distance vector routing and uses hop-by-hop routing approach. Furthermore, AOMDV also finds routes on demand using a procedure of route discovery. The main difference is in the number of routes found in each route discovery. In AOMDV, route request (RREQ) transmission from the source towards the destination establishes multiple reverse paths both at intermediate nodes as well as the destination. Multiple route reply (RREPs) traverse these reverse paths back to form multiple forward paths to the destination at the source and intermediate nodes. Note that AOMDV also provides intermediate nodes with alternate paths as they are found to be useful in reducing route discovery frequency [4]. AOMDV relies on the routing information already available in the underlying AODV algorithm, thereby limiting the overhead incurred in discovering multiple paths. It does not employ any special control packets. In reality, extra RREPs and RERRs for multipath discovery and maintenance along with a few extra fields in routing control packets (i.e., RREQs, RREPs, and RERRs) constitute the only additional overhead in AOMDV relative to AODV [5].

\subsubsection{Ant Colony Optimization}

Ant Colony optimization falls into a class of biologically inspired algorithms that have recently been developed. To name a few, the techniques of Particle Swarm optimization [6] and Bacterial Foraging [7] have been inspired by natural phenomenon. The Ant Algorithm mimics the behavior of ants in nature while they are searching for food. Particle swarm optimization is inspired by the behavior of flocks of birds as they fly in search of food. Also, bacterial foraging is another recent algorithm that simulates the behavior of bacteria searching for food. All these techniques are combinatorial in nature and when viewed in the perspective of optimization involve searching for the optimum solution in a given search space. It has been observed that when these patterns which are observed in nature, are applied to complex engineering problems, they provide good solutions.

These nature inspired techniques share a common characteristic, the whole information about the state of the system is contained not in a single entity, but rather some part of the information is stored in many of the entities. This constitutes swarm intelligence where decisions made by an individual by processing information from not only itself but also other entities. The mechanism of communication of information and the processing of that information is actually where the techniques differ. This prototype forms a great fit for the MANET environment, since it is a decentralized network, in which each node contains only a part of the information about the state of the network [1].

\section{LITERATURE REVIEW}

This section explores some of the researches done on Routing in MANET using Ant Colony Optimization by various researchers in previous years.

In 2013, Aws Kanan et al. [8] applied the Ant Colony evolutionary optimization technique to the routing problem, where more of those desirable properties can be implied in the guided probabilistic choice of paths. Simulations of a routing based on the biological system referred to as Ant Colony Optimization (ACO) were conducted, taking into account several factors to analyze its adaptive nature. Scenarios with high degrees of node mobility showed that the performance of the algorithm in terms of average end-to-end delay and success rate was not degraded.

In 2011, Ma Lin et al. [9] presented an effective congestion elusion scheme explicitly based on ant colony algorithm for mobile ad hoc networks, which explored the optimal route between two nodes promptly and in the meantime forecast congestion state of the link. Accordingly, a new path was found rapidly to have the flow spread around to relieve the congestion state. Results showed that comparing with OLSR, the scheme greatly reduced the packet loss ratio and the average end-to-end delay, thus stating that it makes use of networking resource effectively.

In 2009, Vahide Rajabi Zanjan, et al. [10] presented an algorithm called AAODV for mobile ad hoc networks inspired by Ant Colony Optimization algorithms. It was proposed by maintaining the nature of the Ad hoc On-demand Distance Vector (AODV) routing through the use of Ant Colony Optimization. It maintained the on demand nature of AODV but worked in an adaptive manner. It did not send any Route Request packet that AODV does. Rather it identified its paths with the feedback of previously traveled packets and updated and maintained the paths accordingly. It was probabilistic and adaptive technique that can change its route with the change of network topology by learning the environment. Simulations show that the AAODV algorithm provided better performance in average delay, bandwidth overhead, throughput, and packet loss than the AODV algorithm.

\section{ABOUT THE PROBLEM}

This section discusses about the current problem issues and challenges in MANETs.

\subsection{Routing}

The task of routing is particularly hard in MANETs. Due to the ad hoc and dynamic nature of these networks, the topology can transform continuously, and paths between sources and destinations that were initially efficient can quickly become inefficient or ineffective. This means that routing information should be updated more regularly than in conventional wired telecommunication networks. Though, this can be a problem in MANETs, because they typically have limited bandwidth and node resources, and make use of perhaps unreliable wireless communication channels. Therefore, new routing algorithms are needed, which can give adaptivity in an efficient and robust way [11].

There are various routing algorithms defined and used in MANETs lying in the categories either table-driven or ad-hoc on demand source routing. Some of the efficient ones are AODV in single path routing and AOMDV in multipath routing. Many challenging issues related to routing are handled by many researchers in AODV and AOMDV. 
But as AODV is single path routing algorithm there are no chances of sending the data packets to more than one route. So the problem of load balancing and packet dropping increases due to the changing ad-hoc nature of MANET. As a solution, AOMDV algorithm can be used for sending data packets to multiple paths which can solve the disadvantages of AODV algorithm. This is done applying Ant Colony Optimization in AOMDV.

\subsection{Quality of Service (QoS)}

QoS is also a difficult task for the network developers, because the topology of an ad hoc network is constantly changes. Reserving resources and sustaining a definite quality of service, while the network condition continuously changes, is very challenging [12].

Quality of Service (QoS) refers to a set of service requirements that needs to be met by the network while transporting a packet stream from a source to its destination [13]. Informally, it refers to the probability of a packet passing between two points in the network. The network is estimated to guarantee a set of quantifiable pre-specified service attributes to the users in terms of end-to-end performance, such as bandwidth, delay, probability of packet loss, delay variance (jitter), power consumption etc. The wireless communication was originally developed for military use, because of its installation, ease of mobility and flexibility; later on it was made available to inhabitant use also. With the increasing demand and penetration of wireless services, wireless network users now expect quality of service and performance equivalent to what is available from fixed networks. Several factors that influence QoS of wireless network include:

\subsubsection{Throughput of Network}

It represents the total number of bits (in bits/sec) forwarded from wireless LAN layers to higher layers in all WLAN nodes of the network.

\subsubsection{Retransmission Attempts}

It represents total number of retransmission attempts by all WLAN MACs in the network until either packet is successfully transmitted or it is discarded as a result of reaching short or long retry limit.

\subsubsection{Data Dropped}

It is the data dropped due to unavailability of access to medium.

\subsubsection{Medium Access Delay}

It includes total of contention and queuing delays of the data.

\subsection{Congestion}

In a network with shared resources, where multiple senders contend for link bandwidth, it is necessary to alter the data rate used by each sender such that it does not overload the network. Packets that turn up at a router and cannot be forwarded are dropped, thus an excessive amount of packets arriving at a network blockage leads to many packet drops. These dropped packets might already have travelled a long way in the network and hence consumed considerable resources. Additionally, the lost packets often cause retransmissions which mean that even more packets are sent into the network. Consequently network congestion can severely decline network throughput. If appropriate congestion control is not performed it can lead to congestion collapse of the network, where roughly no data is successfully delivered [14].

\section{PROPOSED WORK}

The central idea of this paper surrounds the application of Ant Colony Optimization to the problem of MANETs. The proposed algorithm is based on Ad-Hoc On-Demand Multipath Routing Protocol (AOMDV) and Ant Colony Optimization (ACO).

\subsection{Ant Colony Optimization in Routing}

ACO routing algorithms take inspiration from the behavior of ants in nature and from the related field of ACO to solve the problem of routing in communication networks. The main source of inspiration is found in the ability of certain types of ants (e.g. the family of Argentine ants Linepithema Humile) to find the shortest path between their nest and a food source using a volatile chemical substance called pheromone. Ants traveling between the food source and the nest leave traces of pheromone as they move. They also preferentially go in the direction of high pheromone intensities. Since shorter paths can be completed faster, they receive higher levels of pheromone earlier, attracting more ants, which in turn lead to more pheromone. This positive reinforcement process allows the colony as a whole to converge on the shortest path. This forms the basis of most of the work in the field of ACO [11].

\subsection{Proposed Ant-AOMDV Algorithm}

The proposed algorithm is based on AOMDV protocol and ant as an agent carrying data packets. The main idea behind the proposed algorithm is that nodes in the network periodically and asynchronously send out artificial ants towards possible destination nodes of data. These ant agents are small control packets, which have the task to find a path towards their destination and gather information about it. Like ants in nature, artificial ants follow and drop pheromone. This pheromone takes the form of routing tables maintained locally by all the nodes of the network. They indicate the relative quality of different routes from the current node towards possible destination nodes. Ants normally take probabilistic routing decisions based on these pheromone tables, giving a positive bias to routes of higher pheromone concentration, in order to balance investigation and utilization of routing information. Often, the tasks of following and updating pheromone are split between a forward and backward ant, whereby the forward ant finds a path towards the destination and the backward ant travels back over the path to update pheromone tables. The result of the constant ant sampling process is the routing information in the pheromone tables, which is used to forward data. This can again be done deterministically following the path with the highest pheromone level. The steps of Ant-AOMDV algorithm are as follows:

1. Set the sender.

2. Set the receiver.

3. Set the nodes.

4. Broadcast Route Request Packets (RREQ) using ant agent.

5. Create pheromone table for all nodes.

6. Find out pheromone value for each node.

7. Send data according to pheromone tables.

8. Analyze the performance of routing behavior of ant technique. 


\section{SIMULATION AND RESULTS}

Implementation of Ant-AOMDV is done in Cygwin and NSAllinone-2.34. Simulation environment is as follows:

Table 1. Environment variables and its associated values used in Experiment

\begin{tabular}{|l|l|l|}
\hline No. & Parameter Variables & Associated Values \\
\hline 1 & Simulation Tool & $\begin{array}{l}\text { Cygwin+Ns-Allinone- } \\
2.34\end{array}$ \\
\hline 2 & Propagation & TwoRayGround \\
\hline 3 & MAC type & Mac/802_11 \\
\hline 4 & Interface Queue & Queue/DropTail \\
\hline 5 & Antenna Type & Omni Direction \\
\hline 6 & $\begin{array}{l}\text { Max. Packet in Interface } \\
\text { Queue }\end{array}$ & 50 \\
\hline 8 & Total Number of Nodes & 20 \\
\hline 7 & Base Protocol & AOMDV \\
\hline 9 & Optimization Algorithm & Ant Colony Optimization \\
\hline 10 & X dimension of Topography & 800 \\
\hline 11 & Y dimension of Topography & 600 \\
\hline 12 & Simulation Time & 100 s \\
\hline
\end{tabular}

For comparison of Ant-AOMDV, there is a need of an ant based algorithm in which pheromone table is generated. But, the entire ant based algorithms made in past are without pheromone table generation. For this purpose, first an ant based single path routing algorithm named Ant-AODV was made. To the best of the knowledge, pheromone table generation has been done in wired environment but in wireless environment it is not yet done. The pheromone table for the proposed Ant-AOMDV is as shown below in Table 2.

Performance Analysis of Ant-AOMDV is done with AntAODV in terms of overall summary of various parameters like Packet Delivery Fraction (PDF), Network Routing Load (NRL), Number of Packets Dropped, Number of sent packets, number of received packets and number of route request packets sent (RREQ). Comparison table of all these parameters are shown below in Table 3 .

On the basis of the parameters from Table 3 necessary graphs for comparison of AODV-ANT and AOMDV-ANT are generated.
Table 2. Pheromone Table of Node 1 for AOMDV-ANT

\begin{tabular}{|r|r|}
\hline Next Hop & Pheromone Value \\
\hline 0 & 0.00481 \\
\hline 2 & 0.01442 \\
\hline 3 & 0.01923 \\
\hline 4 & 0.02404 \\
\hline 5 & 0.02885 \\
\hline 6 & 0.03365 \\
\hline 7 & 0.03846 \\
\hline 8 & 0.04327 \\
\hline 9 & 0.04808 \\
\hline 10 & 0.05288 \\
\hline 11 & 0.05769 \\
\hline 12 & 0.0625 \\
\hline 13 & 0.06731 \\
\hline 14 & 0.07212 \\
\hline 15 & 0.07692 \\
\hline 16 & 0.08173 \\
\hline 17 & 0.08654 \\
\hline 18 & 0.09135 \\
\hline 19 & 0.09615 \\
\hline
\end{tabular}

Table 3. Performance Analysis

Overall Performance analysis

\begin{tabular}{|l|r|r|}
\hline \multicolumn{1}{|c|}{ Parameter } & $\begin{array}{c}\text { AODV- } \\
\text { Ant }\end{array}$ & \multicolumn{1}{c|}{$\begin{array}{c}\text { AOMDV- } \\
\text { Ant }\end{array}$} \\
\hline SEND & 5758 & 6694 \\
\hline RECV & 4870 & 6325 \\
\hline ROUTINGPKTS & 5651 & 4088 \\
\hline PDF & 84.58 & 94.49 \\
\hline NRL & 1.16 & 0.65 \\
\hline No. of dropped data (packets) & 821 & 364 \\
\hline
\end{tabular}

The graphs of number of data packets received, sent, dropped and route request packets (RREQ) sent are shown in the Figures below:

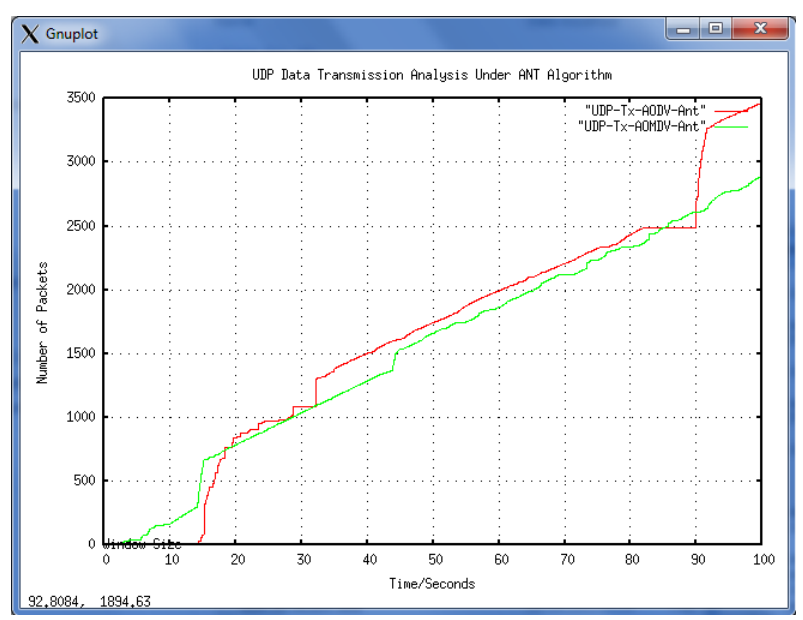

Fig 1: UDP Transmission analysis under ANT Algorithm 


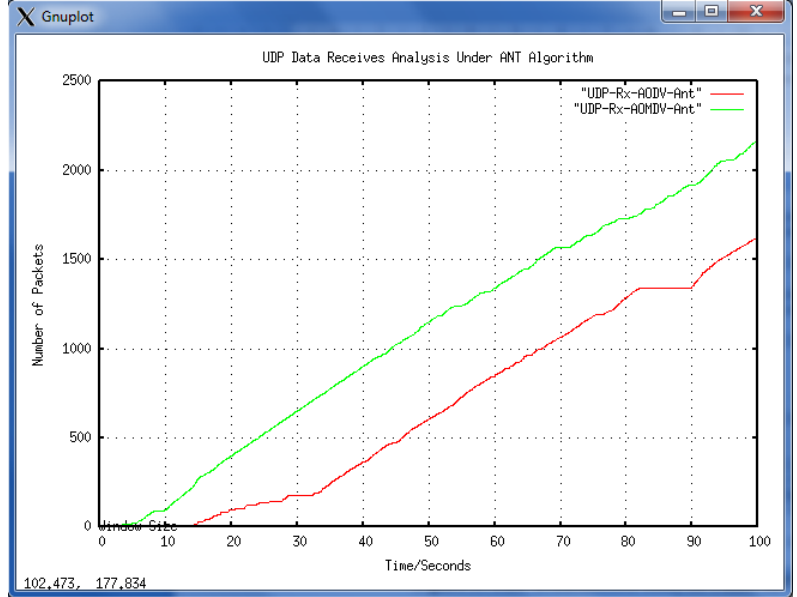

Fig 2: UDP Receiving analysis under ANT Algorithm

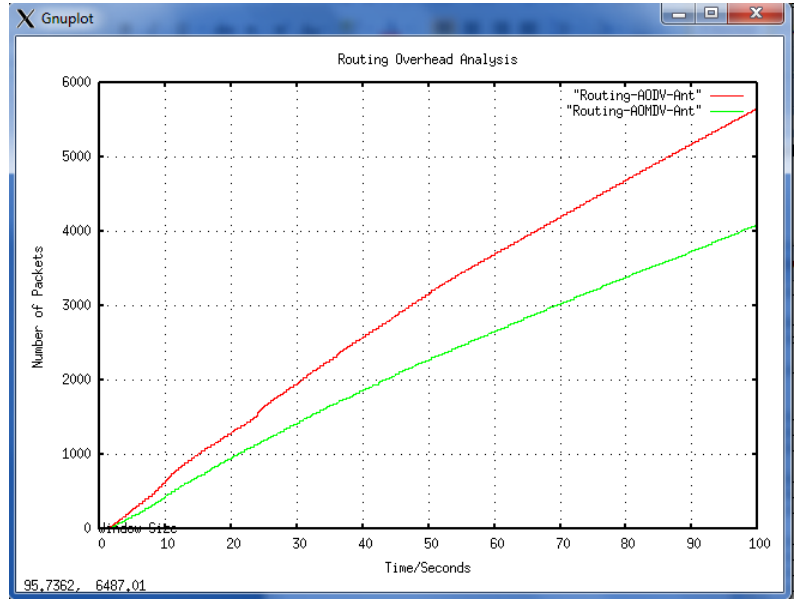

Fig 3: Routing Overhead Analysis

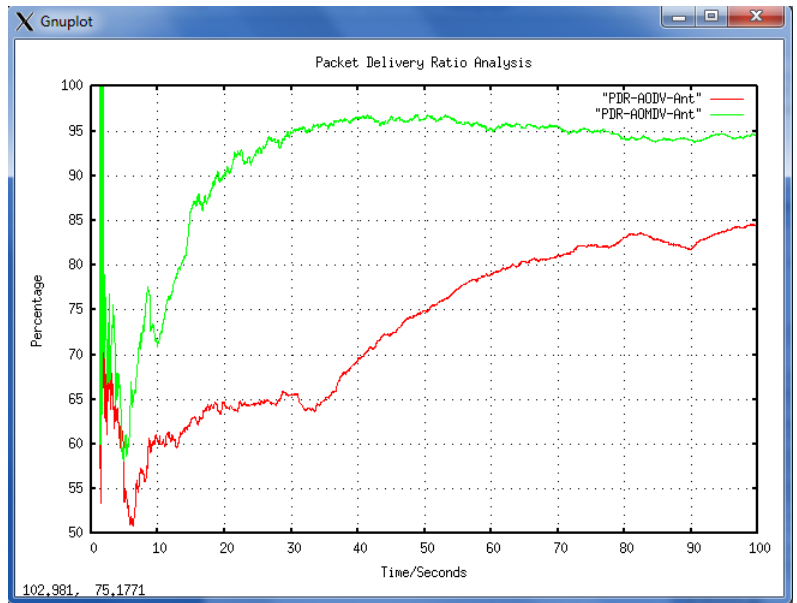

Fig 4: Packet Delivery Ratio Analysis

\section{CONCLUSION AND FUTURE WORK}

This paper presents an ant colony optimization and adhoc ondemand multipath distance vector (AOMDV) based routing protocol that can select stable multiple transmission paths dynamically through regular updating of the pheromone table. It can be seen that the number of dropped packets is more in
AODV-Ant compared to AOMDV-Ant and thus congestion is increased. In AOMDV-Ant multipath routing is done and it sees all possible routes. So, packets can be sent to all the selected paths and thus helps in load balancing and routing overhead is also minimized which is shown with the help of parameters Network Routing Load (NRL) and Routing Overhead respectively. The other performances such as Packet Delivery Fraction (PDF), number of sent packets, number of received packets and number of route request packets sent (RREQ) also shows a vast improvement in AOMDV-Ant. Thus, the proposed AOMDV-Ant can be considered as one of the novel algorithm generating pheromone table efficiently and thus improving other performance measures.

Further work can be done on the implementation to improve the algorithm by analyzing congestion control mechanism through TCP and UDP. Investigations include experiments with high network load. Additionally, analysis of the maintenance of the pheromone concentration is needed. There are diverse ways to manipulate the pheromone concentration on the edges, which manipulate the performance of the routing algorithm.

\section{ACKNOWLEDGMENTS}

We would like to thank the experts who have contributed towards the development of this paper.

\section{REFERENCES}

[1] Subodh M. Iyengar, Soumya N. Pattnaik (2010), "Solving the MANET Routing Problem using Ant Colony Algorithm", B.Tech Thesis, ethesis.nitrkl.ac.in/1682/1/BtechThesis.pdf, National Institute of Technology Rourkela, Department of Computer Science and Engineering.

[2] Donatas Sumyla (2006), "Mobile Ad-Hoc Networks (manets)", Technical Report.

[3] "Ad hoc On-Demand Distance Vector Routing", Wikipedia encyclopedia, http://en.wikipedia.org/wiki/ Ad_hoc_On-Demand_Distance_Vector_Routing.

[4] Nasipuri A, Castaneda R, Das SR (2001) "Performance of multipath routing for on-demand protocols in mobile ad hoc networks". ACM/Kluwer Mobile Networks and Applications (MONET), 6(4):339-349.

[5] Mahesh K. Marina, Samir R. Das (2006) "Ad hoc ondemand multipath distance vector routing", Wireless Communications And Mobile Computing, Published online in Wiley InterScience (www.interscience.wiley.com), 6:969-988.

[6] J. Kennedy and R. Eberhart (1995), "Particle swarm optimization", In Proceedings of IEEE International Conference on Neural Networks, IV, 1942-1948.

[7] K. Passino (2002), "Biomimicry of bacterial foraging for distributed imization and control", Control Systems Magazine, IEEE, 22(3):52 -67.

[8] Aws Kanan, Taisir Eldos, Mohd Alkahtani (2013), "Mobile Ad Hoc Networks Routing Using Ant Colony Optimization", World of Computer Science and Information Technology Journal (WCSIT), ISSN: 22210741 3(6):105-109. 
[9] Ma Lin, Xu Yubin, Meng Weixiao (2011), "An Ant Colony Based Congestion Elusion Routing scheme for MANET", Proceedings published in IEEE Globecom 2011.

[10] Vahide Rajabi Zanjani, Abolfazl Toroghi Haghighat (2009), "Adaptive Routing In Ad Hoc Wireless Networks Using Ant Colony Optimization", International Conference on Computer Technology and Development, Proceedings published in IEEE, 40-45.

[11] Gianni A. Di Caro, Frederick Ducatelle, Luca M. Gambardella (2008), "Ant Colony Optimization for Routing in Mobile Ad Hoc Networks in Urban Environments", Technical Report No. IDSIA-05-08, Dalle Molle Institute for Artificial Intelligence Galleria 2, 6928 Manno, Switzerland.
[12] T. Chen, M. Gerla, and J. T. Tsai (1997), "QoS Routing Performance in a Multi-Hop, Wireless Network," Proceedings published in. IEEE ICUPC ' 97.

[13] R. Ramanathan and M. Steenstrup (1998), "Hierarchically Organized, Multihop Mobile Wireless Networks for Quality of Service Support," Mobile Network and Apps, Vol.3:101-19.

[14] Christian Lochert, Bjorn Scheuermann, Martin Mauve (2007), "A Survey on Congestion Control for Mobile Ad-Hoc Networks", Wiley Wireless Communications and Mobile Computing, 7(5):655-676. http://www.interscience.wiley.com. 